\title{
Meteorological Risk Analysis of Port Crane Operations and Warning Managements
}

\author{
Feng Wang ${ }^{1,2}$, Qiang Long ${ }^{1}$, Chang Wang ${ }^{1}$, Linlin Jiang ${ }^{3}$ \\ ${ }^{1}$ Caofeidian Industrial District Meteorological Bureau, Tangshan 063015, China \\ ${ }^{2}$ Tangshan Meteorological Bureau, Tangshan 063000, China \\ ${ }^{3}$ Petro China Jidong Oilfield Zhida Company, Tangshan 063200, China
}

\section{港口塔吊作业气象风险分析及预警管理}

\author{
王 锋 ${ }^{1,2}$, 龙 强 ${ }^{1}$, 王 申 玚 $^{1}$, 蒋琳琳 ${ }^{3}$ \\ ${ }^{1}$ 唐山市曹妃甸工业区气象局, 唐山 063015, 中国 \\ 2 唐山市气象局, 唐山 063000 , 中国 \\ 3 冀东油田志达公司, 唐山 063020, 中国
}

\begin{abstract}
s
With the rapid development of China's marine economy and the port economy, involved in the work of the security problem is becoming more and more prominent, especially the crane operation risk relates to the port operation, which gets more attention. The operational risk and the early warning and scheduling should be carried out in two aspects of technology and management. The relationship between aerodynamic roughness $z_{0}$ and average wind speed of 10 meters above ground are investigated by using the data from 100-meter-high wind energy tower, which located in the north shore of the Bohai Bay. Besides, a fixed and quantitative wind load forecasting model about port cranes is designed for the requirements of weather service about security operations in ports. The results indicate that two models which based on the relationship between $\mathrm{z} 0$ and average wind speed of 10 meters are available, and it is more suitable for risk forecasting, which can be used as a reliable basis for port crane meteorological risk warning.
\end{abstract}

Keywords: aerodynamic roughness; wind load; risk; warning; management

\section{摘要}

随着我国海洋经济和港口经济的迅速发展, 涉 海作业的安全问题越来越突出, 特别是涉及港 口运营的塔吊作业风险得到了更多的重视, 正 确预计作业风险并对此进行预警调度应从技 术和管理两个方面进行。利用渤海湾北岸 $100 \mathrm{~m}$ 风能塔风观测资料, 研究了空气动力学 粗粘度 $z_{0}$ 和地面 $10 \mathrm{~m}$ 风速之间的关系, 并针 对港口安全运营调度精细化气象服务保障需 求, 设计了一种港口塔吊定点、定量水平风荷 载的预报模型。研究结果显示基于 $z_{0}$ 和地面 $10 \mathrm{~m}$ 风速之间的关系建立的定点、定量风荷载 预报模型正确可行, 更适用于风险预警业务, 可作为港口塔吊气象风险预警的可靠依据。

关键词: 空气动力学粗粘度; 风荷载; 风险; 预警; 管理

1. 引言

近年来我国海洋经济发展取得了长足进步, 社

${ }^{1}$ 作者简介: 王锋 (1962-), 男, 高级工程师, 主要从事海洋预报预警业务管理, 通讯邮箱:q_loong@126.com 
Risk Analysis and Crisis Response in Big Data Era (RAC-16)

会经济效益显著提高。然而, 与海洋经济增长 形成鲜明对比的是我国海洋灾害影响却更加 严重, 中国海洋灾害公报、中国海洋灾害公报 的统计结果触目惊心。大风、大浪、海雾、台 风等极端天气事件已经成为侵蚀我国海洋经 济成果的主要自然灾害。

港口经济的迅速发展带来的港口安全问 题得到了越来越多的关注。由于塔吊较高、风 荷载大, 对垂直梯度风敏感, 作业风险预报不 同于常规天气预报, 当前简单的地面风预测并 不能满足风险预测需求, 这就要求在不同天气 形势下的塔吊整体风荷载有一个有效的预测 方法, 并基于此进行风险预警管理, 也就是说 其涉及到了业务技术和风险管理两个方面 ${ }^{[1]}$ 。

高层构筑物在水平风荷载作用下的结构 分析和设计引起了研究人员和工程师越来越 多的重视 ${ }^{[2-3]}$ 。对于垂直于建（构）筑物表面 上的风荷载标准值的计算, GB50009-2001 《建筑结构荷载规范》详述了相关说明和规定 [4]。目前对风荷载的相关技术规定和科学研究 大多停留在设计阶段, 仅作工程项目风险评估 使用 ${ }^{[5]}$ 。然而, 工程项目落地并投入使用后, 构筑物在日常的运营作业中所面临的情况要 复杂一些 ${ }^{[6-7]}$, 不同地区和下垫面条件下的相 关指标和参数不一, 将参数统一或归类所获得 的风荷载阈值并不能满足安全管理人员对了 解构筑物承受实时风荷载情况的需求, 也无法 实现对未来风向、风速条件下构筑物所承受风 荷载的风险预报。因此, 设计一种便捷可用的 构筑物实时水平风荷载计算模型对作业的安 全运营保障具有重要的现实意义。文中以渤海 湾北岸曹妃甸港为设计案例, 通过分析计算风 能塔观测资料, 获取空气动力学粗糙度特征, 推算垂直梯度风, 并基于伯努利方程, 对接气 象数值预报产品设计了一种港口塔吊水平风 荷载的实时计算和预报预测模型, 并基于研究 成果提出了预警管理思路, 以期为港口安全作 业风险管理提供借鉴。

\section{2. 技术方法}

研究所用数据来自建在渤海湾北岸的 100 $\mathrm{m}$ 风能塔, 共有 5 层观测平台。风能塔所在的 位置如图 1 所示。按照方向划分, $135^{\circ} \sim 315^{\circ}$ 为海-陆风方向 (即风由海面吹向陆地), $0^{\circ} \sim$ $134^{\circ}$ 和 $316^{\circ} \sim 360^{\circ}$ 为陆-海风向 (即风由陆地 吹向海面)。选取了 2012 年 6 月至 2013 年 5 月共计 12 个月 (4 个季节) 的风速、风向等 观测数据, 数据时间分辨率为 $1 \mathrm{~h}$ 。

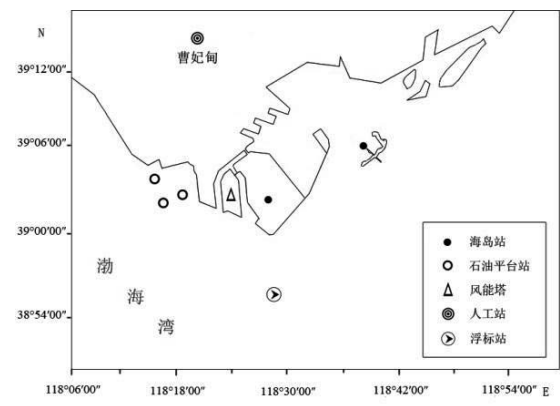

图 1 渤海湾北岸相关观测站点的分布

\section{1 空气动力学粗楉度 $\mathrm{z}_{0}$}

计算空气动力学粗䊁度常用的方法有对数廓 线拟合法、阻力法、质量守恒法、压力中心法 以及无因次化风速法。文中主要采用的是阻力 法。

$$
\sigma_{\mathrm{u}} / U\left(z_{n}\right)=\mathrm{C}_{\mathrm{u}} \mathrm{kln}^{-1}\left(z_{n} / z_{0}\right)
$$

式中, $\sigma_{u}$ 为常数; $U\left(z_{n}\right)$ 为高度 $z_{n}$ 处的风速; $k$ 是卡门常数, 一般取 $0.4 ; \mathrm{C}_{\mathrm{u}}$ 也为常数, 和测 风仪器有关; $z_{n}$ 具有长度量纲。上式适用于 $\mathrm{U}(\mathrm{z})>4.0 \mathrm{~m} / \mathrm{s}$ 的情况。根据数据实际情况和所 采用的方法要求, 需要对测风塔的观测数据进 行质量控制。

采用不同高度处的风速带入上式, 做商即 可消去相关常数, 获得空气动力学粗楉度 $\mathrm{z}_{0}$ 的值

$$
z_{0}=\exp \left[\frac{U(z) \ln z_{n}-U\left(z_{n}\right) \ln z}{U(z)-U\left(z_{n}\right)}\right]
$$

由于气象学地面风观测是位于 $10 \mathrm{~m}$ 高度处, 数值预报产品也是 $10 \mathrm{~m}$ 高度处风速, 结合研 究应用需求, 此次确定 $\mathrm{z}$ 为 $10 \mathrm{~m}, \mathrm{U}(\mathrm{z})$ 即为 $10 \mathrm{~m}$ 高度处的风速。

为进一步分析空气动力学粗粘度随季节、 风向及下垫面的变化及不同计算方法的适用 情况, 在 $\mathrm{z}_{0}$ 特征分析部分增加了对数廓线拟合 计算方法。对数廓线拟合法需要测得 3 个或 3 个以上高度的风速, 用最小二乘回归所得的风 
Risk Analysis and Crisis Response in Big Data Era (RAC-16)

速资料为

$$
U\left(z_{n}\right)=\mathrm{a}+\mathrm{b} \ln z_{n}
$$

按照粗粘度的定义, 令式 (3) 中 $U\left(z_{n}\right)=0$ 即可得空气动力学粗糙度 $\mathrm{z}_{0}$

$$
z_{0}=\exp (-\mathrm{a} / \mathrm{b})
$$

\section{2 塔吊水平风荷载计算模型}

风压是垂直于气流方向的平面单位面积所受 到的风的压力, 根据伯努利方程可得出基本风 -压关系 ${ }^{[8]}$ 。边界层内港口塔吊水平风荷载的实 时计算可忽略近地层空气密度的变化影响, 标 准状态下用风速估计风压的通用公式 ${ }^{[9]}$ 为

$$
w_{p}=U\left(z_{n}\right)^{2} / 1600
$$

式中 $\mathrm{w}_{\mathrm{p}}$ 为风压。根据式 (3) 则可以计算出 $\mathrm{z}_{\mathrm{n}}$ 高度处 $\mathrm{ds}$ 面积的风荷载 $\mathrm{F}\left(\mathrm{z}_{\mathrm{n}}\right)$

$$
F\left(z_{n}\right)=w_{p} \cdot d s=U\left(z_{n}\right)^{2} \cdot d s / 1600
$$

将塔吊机身视作矩形平面, 便有 $\mathrm{ds}=\mathrm{L} * \mathrm{dz}$, $\mathrm{L}$ 为塔吊机身的宽。对式 (4) 采用垂直高度 上的积分即可得塔吊水平风荷载 Fc

$$
F_{c}=\sin \theta \int_{0}^{H} \frac{U\left(z_{n}\right)^{2} L}{1600} d z_{n}
$$

式中, $\mathrm{H}$ 为塔吊的高度, $\theta$ 为风向和塔吊夹角。

由式 (1)、(7) 可知, 实现垂直塔臂水平 风荷载 $\mathrm{F}_{\mathrm{c}}$ 的计算, 主要是研究分析空气动力 学粗䊁度 $\mathrm{z}_{0}$ 的特征和计算方法。

\section{3 预测模型构建}

\section{1 空气动力学粗糙度 $\mathrm{z}_{0}$ 与风速的变化关系}

利用式 (2) 计算得到在海-陆风条件下的空气

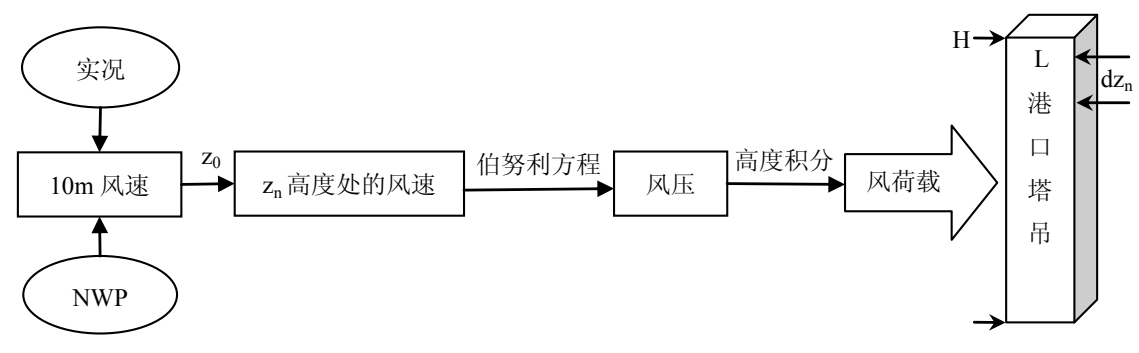

图 3 港口塔吊风荷载计算模型框架
动力学粗䊁度 $\mathrm{z}_{0}$ 的平均值是 $0.59 \mathrm{~m}$, 陆-海风 条件下的平均值是 $0.20 \mathrm{~m}$ 。为了更加准确地获 取不同风速下的粗鋉度值, 分析了 $10 \mathrm{~m}$ 处风 速和空气动力学粗粘度的关系, 并根据数值分 布特征对粗精度值和地面 $10 \mathrm{~min}$ 平均风速做 了公式拟合, 如图 2 所示。
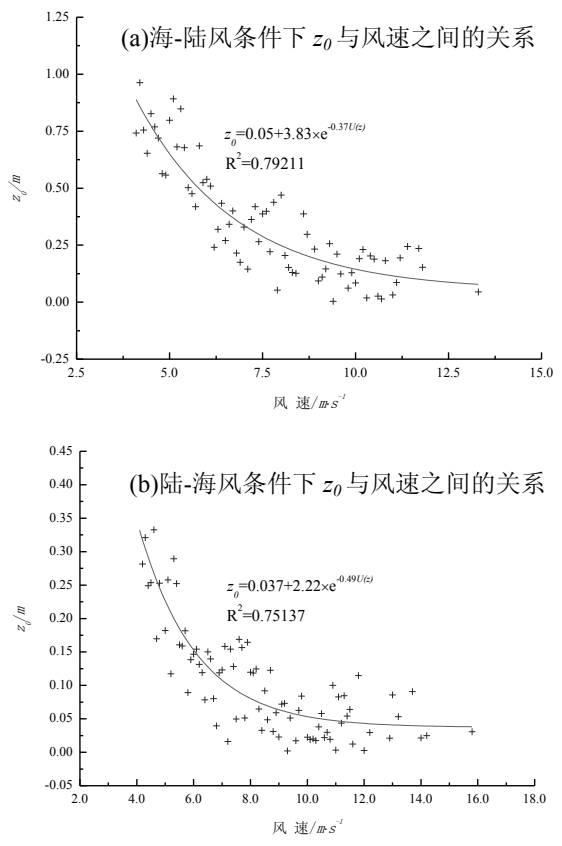

图 2 海-陆风和陆-海风条件下 $z_{0}$ 与风速间的关系

\section{2 基于粗粘度 $\mathrm{z}_{0}$ 的风荷载计算模型}

港口塔吊水平风荷载的实时和预报计算模型 的基本框架如图 3 所示。 
Risk Analysis and Crisis Response in Big Data Era (RAC-16)

综上所述海-陆风条件下的水平风荷载

$$
F_{c}=\frac{\sigma_{\mathrm{u}}{ }^{2} L \sin \theta}{\mathrm{C}_{\mathrm{u}}{ }^{2} \mathrm{k}^{2} 1600} \int_{0}^{H} \ln ^{2} \frac{z_{n}}{0.05+3.83 \times e^{-0.37 U(z)}} d z_{n}
$$

陆-海风条件下的水平风荷载

$$
F_{c}=\frac{\sigma_{\mathrm{u}}{ }^{2} L \sin \theta}{\mathrm{C}_{\mathrm{u}}{ }^{2} \mathrm{k}^{2} 1600} \int_{0}^{H} \ln ^{2} \frac{z_{n}}{0.037+2.22 \times e^{-0.49 U(z)}} d z_{n}
$$

消减参数后海-陆风条件下的水平风荷载

$$
F_{c}=\frac{L \sin \theta}{1600} \int_{0}^{H}\left(\frac{\ln \left[\left(0.05+3.83 \times e^{-0.37 U(z)}\right) / z_{n}\right]}{\ln \left[\left(0.05+3.83 \times e^{-0.37 U(z)}\right) / z\right]} U(z)\right)^{2} d z_{n}
$$

消减参数后陆-海风条件下的水平风荷载

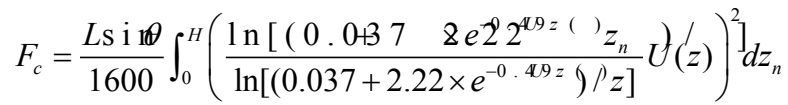

水平风荷载最终变为关于 $z_{n}$ 的积分

$$
F_{c}=\frac{L \cdot \sin \theta \cdot U^{2}(z)}{1600 \cdot \ln ^{2}\left(z_{0} / z\right)} \int_{0}^{H} \ln ^{2}\left(z_{0} / z_{n}\right) d z_{n}
$$

\section{4. 风险预警应用和管理}

风险预警应用平台设计框架如图 4。

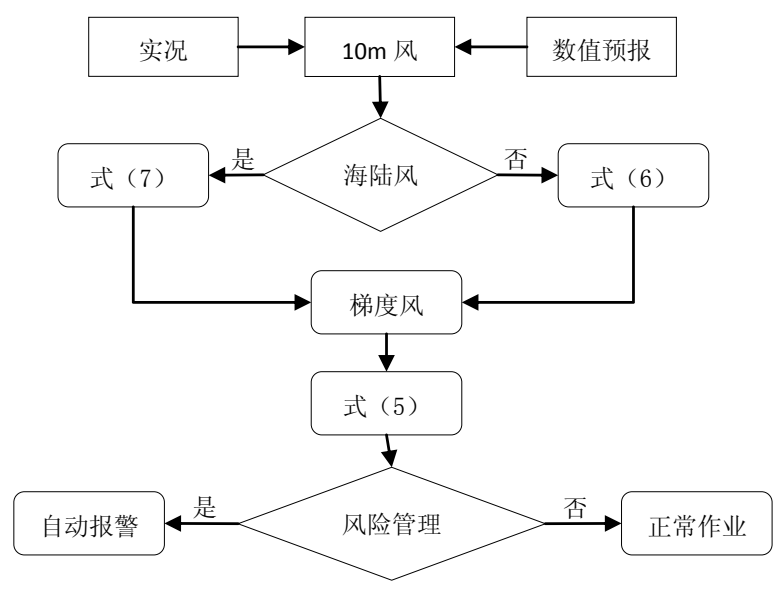

图 4 程序设计框架图

风险管理方面主要是基于以上的计算 结果, 参照项目设计阶段所确定的风荷载國 值，实现不同风条件下的塔吊风险评估，提 前做好作业安排。

为了评估模型的适用性, 选取了 2015
年 8 月的风速实况进行试验。表 1 为渤海湾 北岸曹妃甸港口南北方向上塔吊（偏东方向 作业, 垂直单臂起重机) 的水平风荷载指标, 风等级以平均风速划分。由表可知, 通过该 模型所得的风险警报指标（停止作业指标在 
Risk Analysis and Crisis Response in Big Data Era (RAC-16)

警报指标上增加 1 级）和安全运营调度所设 在忽略塔机高度, 将单臂视作立方体所致。 计的國值基本一致, 整体略偏小, 这是由于

表 1 曹妃甸港口塔吊安全运营调度风等级指标

\begin{tabular}{ccccccccc}
\hline 风向 & $\mathrm{N}$ & $\mathrm{NE}$ & $\mathrm{E}$ & $\mathrm{SE}$ & $\mathrm{S}$ & $\mathrm{SW}$ & $\mathrm{W}$ & $\mathrm{NW}$ \\
\hline 警报指标 & 5 级 & 6 级 & 7 级 & 6 级 & 5 级 & 6 级 & 7 级 & 6 级 \\
停止作业指标 & 6 级 & 7 级 & 8 级 & 7 级 & 6 级 & 7 级 & 8 级 & 7 级 \\
调度设定國值 & 7 级 & 7 级 & 8 级 & 8 级 & 7 级 & 7 级 & 8 级 & 7 级 \\
\hline
\end{tabular}

以上分析结果显示, 调度中心在获取水 平风荷载计算值后，对比停止作业指标，即 可决定作业安排，可有效避免相应风险及其 带来的损失。

对于龙门吊、十字起重机等其他形式的 塔吊，同样适用于该方法，垂直方向的风荷 载仍然采用上述方法, 水平方向上由于不存 在梯度风差异（水平塔臂的垂直高度较小, 忽略垂直方向上风速的不同)，可计算该高 度处的梯度风速, 求得水平塔臂的风荷载

$$
F_{s}=\sin \theta \frac{U(h)^{2} S_{s}}{1600}
$$

式中 $h$ 为水平塔臂所在的高度, $U(h)$ 可通过 (8) 式求得, $S_{s}$ 为水平塔臂的平面面积, 为 固定值。因此, 十字起重机的风荷载 $F=v \cdot\left(F_{c}+F_{s}\right), v$ 为因镂空所致的削减系数, 在 $0 \sim 1$ 之间; 而龙门吊由于有两条垂直塔臂, 整体的水平风荷载 $F=2 F_{c}+F_{s}$ 。

\section{5. 结论}

（1）渤海湾北岸 $\mathrm{z}_{0}$ 具有明显的月变化, 且 地面 $10 \mathrm{~m}$ 风速和 $\mathrm{z}_{0}$ 间存在稳定的指数关系, 计算检验显示所建立的方程稳定可行, 风压 计算模型可用。

（2）港口塔吊安全作业涉及业务技术和风 险管理两大方面, 前者是后者的技术基础, 提供决策依据, 后者是前者的应用深化, 提 供实用的风险预测产品。

（3）文中提出的预测方法计算结果可为调 度决策提供参考, 基于此的预警管理可有效 规避损失风险。

\section{Acknowledgements}

This study was supported by CMA Meteorological key technology integration and application projects (CMAGJ2015M5).

\section{致谢}

本研究得到了中国气象局关键技术集成与 应用项目（CMAGJ2015M5）的支持。

\section{6. 参考文献}

[1] Guo Shujun. The Meteorological Disaster Risk Assessment Based on the Diffusion Mechanism. Journal of Risk Analysis and Crisis Response,2012,2(2):124-130.

[2] 周红波,黄誉.超高层建筑在极端台风气 候下结构及施工安全风险分析及控制 研究.土木工程学报，2014，47(7): 126-135.

[3] 吴角春,张皖华, 叶小刚,等.塔吊倾覆力 矩的计算.科技信息,2007(24):352+374.

[4] GB50009-2001 建筑结构荷载规范. 北京: 中国建筑工业出版社,2006.

[5] 郑永胜,李雪梅,王莹. 风荷载在地质灾害 中的作用.世界地质,2007,26(3):333-337.

[6] 陈晖,曹立华,李安龙,等.埕岛海域浅水 区人工构筑物周边海底地形演化与海 洋灾害地质现象关系研究.海岸工 程,2003,22(3):19-24.

[7] 褚宏宪,原晓军,张晓波,等.石岛新港港 池回淤变化分析. 海岸工 程,2009,28(4):20-29.

[8] 苏志,张瑞波,周绍毅,等. 北部湾沿海基 本风压和阵风风压分析. 热带地理, 2010, 30(2):141-144.

[9] 袁春红,薛桁,杨振斌.建筑风荷载风压计 算中的若干问题.气象,2002:28(1):39-41. 